\title{
A New Exponential-X Family: Modeling Extreme Value Data in the Finance Sector
}

\author{
Zubair Ahmad (D), ${ }^{1}$ Eisa Mahmoudi $\left(\mathbb{D},{ }^{1}\right.$ Rasool Roozegar, ${ }^{1}$ Morad Alizadeh, ${ }^{2}$ \\ and Ahmed Z. Afify \\ ${ }^{1}$ Department of Statistics, Yazd University, P.O. Box 89175-741, Yazd, Iran \\ ${ }^{2}$ Department of Statistics, Faculty of Intelligent Systems Engineering and Data Science, Persian Gulf University, \\ Bushehr 75169, Iran \\ ${ }^{3}$ Department of Statistics, Mathematics and Insurance, Benha University, Benha 13511, Egypt
}

Correspondence should be addressed to Eisa Mahmoudi; emahmoudi@yazd.ac.ir

Received 22 July 2021; Revised 4 October 2021; Accepted 6 October 2021; Published 21 October 2021

Academic Editor: Amer Al-Omari

Copyright (C) 2021 Zubair Ahmad et al. This is an open access article distributed under the Creative Commons Attribution License, which permits unrestricted use, distribution, and reproduction in any medium, provided the original work is properly cited.

In this paper, a family of statistical models, namely, a new exponential- $X$ family is proposed. A subcase of the introduced family, called the new exponential-Weibull (NE-Weibull) model, is studied. The NE-Weibull model is very competent and possesses heavy-tailed properties. The maximum likelihood estimators of its parameters are derived. The consistency and efficiency of these estimators are assessed in a brief simulation study. Finally, the effectiveness of the NE-Weibull distribution is illustrated by modeling real insurance claims data. The practical analysis shows that the NE-Weibull distribution outclassed other distributions and it can be a better choice for modeling data in the finance sector.

\section{Introduction}

The extreme value phenomena such as financial returns and other related events can be modeled effectively by extreme value methods. The heavy-tailed (HT) distributions have been proven to be substantial in modeling HT and extreme value data. Researchers have shown a deep concern in the financial sector to study new HT distributions. Among the applicability of the statistical distributions in the applied area, the HT distributions have received much attention for modeling financial phenomena. Most of the data sets in the financial sector possesses HT behavior with long right tail (see Lane [1]; Cooray and Ananda [2]; Wang et al. [3]; Ahn et al. [4]; Jelenković and Tan [5]; Forbes and Wraith [6]; Guo [7]; Punzo et al. [8]; Bhati and Ravi [9]; Punzo [10]; Ke et al. [11]; Dos Reis et al. [12]; and Niu et al. [13]).

Recently, different copulas and new approaches of introducing HT distribution have been studied due to the importance of HT distributions in the financial sector (for example, Bladt et al. [14]; Tikhomirov [15]; Lugosi and Mendelson [16]; and Yousri et al. [17]). For more information about the usefulness of statistical distributions, one can refer to studies by Ramos et al. [18, 19]; He et al. [20]; Alfaer et al. [21]; Afify et al. [22]; and Al Mutairi et al. [23].

A statistical distribution with SF (survival function), say $\bar{M}(x ; \Delta)=1-M(x ; \Delta)$, is said to be a HT model, if its SF verifies

$$
\lim _{x \rightarrow \infty} \exp (\eta x)[1-M(x ; \Delta)]=\infty,
$$

for all $\eta>0$. For more details, see the study by Resnick [24].

An interesting characteristic of the HT distributions is the regularly varying behavior. A statistical distribution is said to possess the regularly varying behavior, if it satisfies

$$
\lim _{x \longrightarrow \infty} \frac{1-M(q x ; \Delta)}{1-M(x ; \Delta)}=q^{b},
$$

where $b \in\{0, \infty\}$, and it is also known as an index of regular variation.

The statistical models possessing such property are very prominent models for modeling HT phenomena in the financial sector [25]. Furthermore, actuaries are very interested in looking for new flexible HT models (see Nadarajah and Bakar [26]; and Ahmad et al. [27]). 
Alzaatreh et al. [28] proposed a prominent approach called the T- $X$ family method. Let $u(t)$ represent the PDF (probability density function) of $T$, where $T$ is a RV (random variable) belonging to $\left[\delta_{1}, \delta_{2}\right]$ and $-\infty \leq \delta_{1}<\delta_{2}<\infty$. Suppose that $O[H(x ; \Delta)]$ is a function of $H(x ; \Delta)$ of a RV-X and it has the following conditions:

(i) $O[H(x ; \Delta)] \in\left[\delta_{1}, \delta_{2}\right]$

(ii) $O[H(x ; \Delta)]$ is a differentiable function as well as monotonically increasing

(iii) $O[H(x ; \Delta)] \longrightarrow \delta_{1}$ for $\quad x \longrightarrow-\infty \quad$ and $\mathrm{O}[H(x ; \Delta)] \longrightarrow \delta_{2}$ for $x \longrightarrow \infty$

The CDF (cumulative distribution function) of the T- $X$ distributions is

$$
M(x ; \Delta)=\int_{\delta_{1}}^{O[H(x ; \Delta)]} u(t) \mathrm{d} t, \quad x \in \mathbb{R},
$$

where $O[H(x ; \Delta)]$ fulfills the aforementioned conditions. The corresponding PDF of equation (3), say $m(x ; \Delta)$, reduces to

$$
m(x ; \Delta)=\left\{\frac{\partial}{\partial x} O[H(x ; \Delta)]\right\} u\{O[H(x ; \Delta)]\} .
$$

More details about the T- $X$ approach can be found in the work of Ahmad et al. [29]. By implementing the T-X method, the survival distribution family [30] can be obtained via the CDF:

$$
M(x ; \Delta)=1-\int_{\delta_{1}}^{O[\bar{H}(x ; \Delta)]} u(t) \mathrm{d} t, \quad x \in \mathbb{R},
$$

where $\bar{H}(x ; \Delta)=1-H(x ; \Delta)$, representing the SF of $X$.

In this study, a new exponential- $X$ (NE- $X)$ family is proposed based on equation (3). Suppose $T \sim \exp (1)$; then, its respective $\mathrm{CDF}$ is

$$
U(t)=1-\exp (-t), \quad t \geq 0 .
$$

Linking this to equation (6), the PDF is

$$
u(t)=\exp (-t)
$$

By using $u(t)$ and $O[H(x ; \beta, \Delta)]=-\log \left(\left(1-H^{\beta}(x ; \Delta)\right) / e^{H^{\beta}(x ; \Delta)}\right)$ in equation (3), the CDF of the NE- $X$ family is as follows:

$$
M(x ; \beta, \Delta)=1-\left(\frac{1-H^{\beta}(x ; \Delta)}{e^{H^{\beta}(x ; \Delta)}}\right), \quad \beta>0, x, \Delta \in \mathbb{R},
$$

where $H(x ; \Delta)$ is a baseline CDF with parametric space $\Delta \in \mathbb{R}$. Next, in Propositions 1 and 2, we are going to prove that the expression provided in equation (8) is a CDF.

Proposition 1. For the $M(x ; \beta, \Delta)$ defined in equation (8), $\lim _{x \longrightarrow-\infty} M(x ; \beta, \Delta)=0$ and $\lim _{x \longrightarrow \infty} M(x ; \beta, \Delta)=1$.

$$
\begin{aligned}
\lim _{x \rightarrow \infty} M(x ; \beta, \Delta) & =\lim _{x \longrightarrow-\infty}\left\{1-\left(\frac{1-H^{\beta}(x ; \Delta)}{e^{H^{\beta}(x ; \Delta)}}\right)\right\} \\
& =1-\left(\frac{1-H^{\beta}(-\infty ; \Delta)}{e^{H^{\beta}(-\infty ; \Delta)}}\right) \\
& =1-\left(\frac{1-0}{e^{0}}\right)=0,
\end{aligned}
$$

$$
\begin{aligned}
\lim _{x \rightarrow \infty} M(x ; \beta, \Delta) & =\lim _{x \rightarrow \infty}\left\{1-\left(\frac{1-H^{\beta}(x ; \Delta)}{e^{H^{\beta}(x ; \Delta)}}\right)\right\} \\
& =1-\left(\frac{1-H^{\beta}(\infty ; \Delta)}{e^{H^{\beta}(\infty ; \Delta)}}\right) \\
& =1-\left(\frac{1-1}{e}\right)=1 .
\end{aligned}
$$

Proposition 2. The CDF $M(x ; \beta, \Delta)$ is right continuous and differentiable.

Proof.

$$
\frac{\mathrm{d}}{\mathrm{d} x} M(x ; \beta, \Delta)=m(x ; \beta, \Delta)
$$

From the results proved in Propositions 1 and 2, we arrive at the conclusion that the function $M(x ; \beta, \Delta)$ defined in equation (8) is differentiable, right continuous, and a compact CDF. Moreover, taking into account the differentiability property of $M(x ; \beta, \Delta)$ for all $x \in \mathbb{R}$, we have the following theorem.

Theorem 1. Let $\kappa_{1}(x)=e^{H^{\beta}(x ; \Delta)}+H^{\beta}(x ; \Delta)-1$ and $\kappa_{2}(x)=e^{H^{\beta}(x ; \Delta)}$. With the condition $v_{2}(x) \neq 0$, if $\kappa_{1}(x)$ and $\kappa_{2}(x)$ are differentiable, then the quotient $\left(\kappa_{1}(x) / \kappa_{2}(x)\right)$ is differentiable for all $x \in \mathbb{R}$ and

$$
\frac{\mathrm{d}}{\mathrm{d} x}\left[\frac{\kappa_{1}(x)}{\kappa_{2}(x)}\right]=\frac{\kappa_{1}^{\prime}(x) \kappa_{2}(x)-\kappa_{1}(x) \kappa_{2}^{\prime}(x)}{\kappa_{2}^{2}(x)}
$$

Proof. See "Proof of Quotient Rule."

Taking into account the right continuity of $M(x ; \beta, \Delta)$, we have the following theorem.

Theorem 2. If $H(x ; \Delta)$ is a right continuous function, so is $M(x ; \beta, \Delta)$.

Proof. Assume that $\lim _{x \rightarrow a^{+}} H(x ; \Delta)=H(a ; \Delta)$ for all $a \in \sup (H(x ; \Delta))$. Hence, we have

$$
\begin{aligned}
\lim _{x \longrightarrow a^{+}}\left\{e^{H^{\beta}(x ; \Delta)}+H^{\beta}(x ; \Delta)-1\right\} & =e^{H^{\beta}(a ; \Delta)}+H^{\beta}(a ; \Delta)-1, \\
\lim _{x \longrightarrow a^{+}} e^{H^{\beta}(x ; \Delta)} & =e^{H^{\beta}(a ; \Delta)} .
\end{aligned}
$$

Since $\sup (M(x ; \beta, \Delta))=\sup (H(x ; \Delta))$, 


$$
\lim _{x \longrightarrow a^{+}} M(x ; \beta, \Delta)=M(a ; \beta, \Delta),
$$

for each $a \in \sup (M(x ; \beta, \Delta))$. Therefore, $M(x ; \beta, \Delta)$ is right continuous.

As we mentioned earlier that the regularly varying tail behavior (RVTB) is a very crucial property to characterize the HT distributions, now we provide the mathematical treatment of RVTB of the NE- $X$ family.

Theorem 3. If $\bar{H}(x ; \Delta)$ is a regularly varying distribution, so is $\bar{M}(x ; \beta, \Delta)$.

Proof. Suppose $\lim _{x \rightarrow \infty}\left(\left(1-H^{\beta}(b x ; \beta, \Delta)\right) /\left(1-H^{\beta}(x ; \beta\right.\right.$, $\Delta)))=g(b)$ is finite for all $b>0$. Then, using equation (8), we have

$$
\begin{aligned}
\lim _{x \rightarrow \infty} \frac{\bar{M}(b x ; \beta, \Delta)}{\bar{M}(x ; \beta, \Delta)} & =\lim _{x \rightarrow \infty} \frac{1-H^{\beta}(b x ; \Delta)}{1-H^{\beta}(x ; \Delta)} \times \frac{e^{H^{\beta}(x ; \Delta)}}{e^{H^{\beta}(b x ; \Delta)}} \\
& =\lim _{x \rightarrow \infty} \frac{1-H^{\beta}(b x ; \Delta)}{1-H^{\beta}(x ; \Delta)} \times \frac{e^{H^{\beta}(\infty ; \Delta)}}{e^{H^{\beta}(b . \infty ; \Delta)}} .
\end{aligned}
$$

Since $\lim _{x \rightarrow \infty} H(x ; \Delta)=1$, we have

$$
\begin{aligned}
\lim _{x \rightarrow \infty} \frac{\bar{M}(b x ; \beta, \Delta)}{\bar{M}(x ; \beta, \Delta)} & =\lim _{x \longrightarrow \infty} \frac{1-H^{\beta}(b x ; \Delta)}{1-H^{\beta}(x ; \Delta)} \times \frac{e}{e} \\
& =\lim _{x \longrightarrow \infty} \frac{1-H^{\beta}(b x ; \Delta)}{1-H^{\beta}(x ; \Delta)}=g(b),
\end{aligned}
$$

for $b>0$; thus, $\bar{M}(x, \beta, \Delta)$ is a regularly varying distribution.

Linking this to equation (8), the PDF represented by $m(x ; \beta, \Delta)$ is

$m(x ; \beta, \Delta)=\frac{\beta h(x ; \Delta) H^{\beta-1}(x ; \Delta)}{e^{H^{\beta}(x ; \Delta)}}\left\{2-H^{\beta}(x ; \Delta)\right\}, \quad x \in \mathbb{R}$.

The RV with PDF (16) is represented by $X \sim N E-X(x ; \beta, \Delta)$.

The SF, $\bar{M}(x ; \beta, \Delta)$, and HRF (hazard rate function), $w(x ; \beta, \Delta)$, of $X$ are

$$
\bar{M}(x ; \beta, \Delta)=\left(\frac{1-H^{\beta}(x ; \Delta)}{e^{H^{\beta}(x ; \Delta)}}\right), \quad x, \in \mathbb{R},
$$

$$
w(x ; \beta, \Delta)=\frac{\beta h(x ; \Delta) H^{\beta-1}(x ; \Delta)}{1-H^{\beta}(x ; \Delta)}\left\{2-H^{\beta}(x ; \Delta)\right\}, \quad x \in \mathbb{R},
$$

respectively. In the next section, we discuss a subcase of the $\mathrm{NE}-X$ family, called the new exponential-Weibull (NEWeibull) distribution.

\section{NE-Weibull Distribution}

This section deals with the NE-Weibull distribution as a subcase of the NE- $X$ family. Suppose that $X \sim \operatorname{Weibull}(\alpha, \gamma)$ with $\mathrm{CDF}, \quad H(x ; \Delta)=1-\exp \left(-\gamma x^{\alpha}\right)$, and PDF, $h(x ; \Delta)=\alpha \gamma x^{\alpha-1} \exp \left(-\gamma x^{\alpha}\right)$, where $\Delta=(\alpha, \gamma)$. By inserting the CDF of the Weibull model in equation (8), the CDF and PDF of the NE-Weibull distribution take the forms

$$
\begin{aligned}
& M(x ; \beta, \Delta)=1-\frac{1-\left[1-\exp \left(-\gamma x^{\alpha}\right)\right]^{\beta}}{\exp \left\{\left[1-\exp \left(-\gamma x^{\alpha}\right)\right]^{\beta}\right\}}, \quad x \geq 0, \alpha, \gamma, \beta>0, \\
& m(x ; \beta, \Delta)=\frac{\alpha \gamma \beta x^{\alpha-1} \exp \left(-\gamma x^{\alpha}\right)\left[1-\exp \left(-\gamma x^{\alpha}\right)\right]^{\beta-1}}{\exp \left\{\left[1-\exp \left(-\gamma x^{\alpha}\right)\right]^{\beta}\right\}}\left\{2-\left[1-\exp \left(-\gamma x^{\alpha}\right)\right]^{\beta}\right\} .
\end{aligned}
$$

Some possible PDF behaviors for the NE-Weibull distribution are shown in Figure 1. From Figure 1, it is obvious that as the values of $\alpha$ and $\beta$ increase, the NE-Weibull model becomes a HT distribution.

As mentioned above, different PDF shapes of the NEWeibull model for various values of $\beta, \gamma=1$, and $\alpha$ are sketched in Figure 1 . When $\alpha, \beta<1$, the NE-Weibull distribution behaves similar to the exponential distribution $\exp (\gamma)$. For $\beta>1$, the NE-Weibull distribution captures the characteristics of Wei $(\alpha, \gamma)$. The NE-Weibull model, however, has certain benefits over the Weibull distribution. For instance, it has heavier tails than the Weibull distribution and provides closer fit to data in the finance sector.

Some possible HRF behaviors for the NE-Weibull distribution are shown in Figure 2. The NE-Weibull distribution provides increasing, unimodal, decreasing, and bathtub HRF shapes.

\section{Properties}

Here, we provide a concise treatment of the mathematical properties of NE- $X$ distribution. These properties include QF (quantile function), moments, SK (skewness), and KUR (kurtosis). Furthermore, different plots for the SK and KUR are also provided.

3.1. Quantile Function. Suppose $X$ denotes the NE- $X$ family with CDF presented in equation (8); then, the QF of NE- $X$ distributions, $Q(u)$, is

$$
x_{u}=Q(u)=M^{-1}(u)=H^{-1}(t),
$$


where $t$ is the solution of $H(x ; \Delta)+H^{\beta}(x ; \Delta)+\log (1-u)-1$.

3.2. Moments. Now, we introduce the moments of the NE- $X$ distributions which can further be used to obtain other characteristics.

The $r^{\text {th }}$ moment of the NE- $X$ family reduces to

$$
\mu_{r}^{\prime}=\int_{-\infty}^{\infty} x^{r} m(x ; \beta, \Delta) \mathrm{d} x .
$$

By putting equation (16) in (20), we obtain

$$
\begin{aligned}
\mu_{r}^{\prime} & =\beta \int_{-\infty}^{\infty} x^{r} \frac{h(x ; \Delta) H^{\beta-1}(x ; \Delta)}{\exp \left\{H^{\beta}(x ; \Delta)\right\}}\left\{2-H^{\beta}(x ; \Delta)\right\} \mathrm{d} x \\
& =\beta \sum_{i=0}^{n} 2^{1-i}(-1)^{i}\left(\begin{array}{c}
n \\
i
\end{array}\right) \int_{-\infty}^{\infty} x^{r} \frac{h(x ; \Delta) H^{\beta+i \beta-1}(x ; \Delta)}{\exp \left\{H^{\beta}(x ; \Delta)\right\}} \mathrm{d} x \\
& =\beta \sum_{i=0}^{n} \sum_{j=0}^{\infty} 2^{1-i}(-1)^{i+j}\left(\begin{array}{c}
n \\
i
\end{array}\right) \kappa_{r, i, j, \beta},
\end{aligned}
$$

where $\quad \kappa_{r, i, j, \beta}=\int_{-\infty}^{\infty} x^{r} h(x ; \Delta) H^{\beta+i \beta+j \beta-1}(x ; \Delta) \mathrm{d} x$. For $r=1,2,3,4$, we obtain the first four moments of the NE- $X$ family.

Using the expressions of the moments, we obtain the mathematical form of the SK and KUR measures. The SK and KUR of the NE-Weibull distribution can be calculated, respectively, via the expressions

$$
\begin{array}{r}
\mathrm{SK}=\frac{\mu_{3}}{\mu_{2}^{3 / 2}}, \\
\mathrm{KUR}=\frac{\mu_{4}}{\mu_{2}^{2}} .
\end{array}
$$

The effects of $\beta, \alpha$, and $\gamma$ on the SK, KUR, variance, and mean of the NE-Weibull distribution are displayed in Figures 3-5.

\section{Estimation}

Within this section, we derive the maximum likelihood estimators (MLEs) of the NE-Weibull parameters. Consider $x_{1}, x_{2}, \ldots, x_{n}$ as the values of a sample from the NE-Weibull distribution with parameters $\beta$ and $\Delta$. The log-likelihood (LL) function $\ell(\beta, \Delta)$ of the NE-Weibull distribution takes the form

$$
\begin{aligned}
\ell(\beta, \Delta)= & k \log \beta+\sum_{i=0}^{k} \log h\left(x_{i} ; \Delta\right) \\
& +(\beta-1) \sum_{i=0}^{k} \log H\left(x_{i} ; \Delta\right)-\sum_{i=0}^{k} H^{\beta}\left(x_{i} ; \Delta\right) \\
& +\sum_{i=0}^{k} \log \left[2-H^{\beta}\left(x_{i} ; \Delta\right)\right] .
\end{aligned}
$$

The partial derivatives of the LL function are

$$
\begin{aligned}
& \frac{\partial}{\partial \beta} \ell(\beta, \Delta)= \frac{n}{\beta}+\sum_{i=0}^{k} \log H\left(x_{i} ; \Delta\right)-\sum_{i=0}^{k}\left[\log \left(H^{\beta}\left(x_{i} ; \Delta\right)\right)\right] H^{\beta}\left(x_{i} ; \Delta\right) \\
&-\sum_{i=0}^{k} \frac{\left[\log \left(H^{\beta}\left(x_{i} ; \Delta\right)\right)\right] H^{\beta}\left(x_{i} ; \Delta\right)}{2-H^{\beta}\left(x_{i} ; \xi\right)}, \\
& \frac{\partial}{\partial \Delta} \ell(\beta, \Delta)=(\beta-1) \sum_{i=0}^{k} \frac{\partial H\left(x_{i} ; \Delta\right) / \partial \Delta}{H\left(x_{i} ; \Delta\right)}-\beta \sum_{i=0}^{k} \frac{H^{\beta-1}\left(x_{i} ; \Delta\right) \partial H\left(x_{i} ; \Delta\right)}{\partial \Delta} \\
&+\sum_{i=0}^{k} \frac{\partial h\left(x_{i} ; \Delta\right) / \partial \Delta}{h\left(x_{i} ; \Delta\right)}-\beta \sum_{i=0}^{k} \frac{H^{\beta-1}\left(x_{i} ; \Delta\right) \partial H\left(x_{i} ; \Delta\right) / \partial \Delta}{2-H^{\beta-1}\left(x_{i} ; \Delta\right)} . \\
& H(x ; \Delta)+(H(x ; \Delta))^{\theta}+\log (1-u)-1 .
\end{aligned}
$$

Equating $(\partial / \partial \beta) \ell(\beta, \Delta)$ and $(\partial / \partial \Delta) \ell(\beta, \Delta)$ to zero and simultaneously solving them yield the MLEs $(\widehat{\beta}, \widehat{\Delta})$ of $(\beta, \Delta)$.

\section{Simulation Study}

Here, we implement the Monte Carlo simulation approach to address the MLE behavior in estimating the NE-Weibull parameters. The NE-Weibull distribution can be simulated by using equation (8). Let $U$ follow the standard uniform distribution; hence, the quantile function reduces to
The simulation is done for (i) $\alpha=0.5, \beta=1.2, \gamma=1$, (ii) $\alpha=1.2, \beta=0.8, \gamma=0.9$, and (iii) $\alpha=0.8, \beta=0.5, \gamma=1.1$.

The simulation results are obtained by utilizing the $R$ software with the algorithms (root Solve) and "LBFGS - B" with optim. The results obtained are based on $K=500$ replications for samples of size $k$, where $k=10,20, \ldots, 500$. Statistical tools such as biases and mean square errors (MSEs) are obtained as assessing tools. These tools are calculated as follows: 

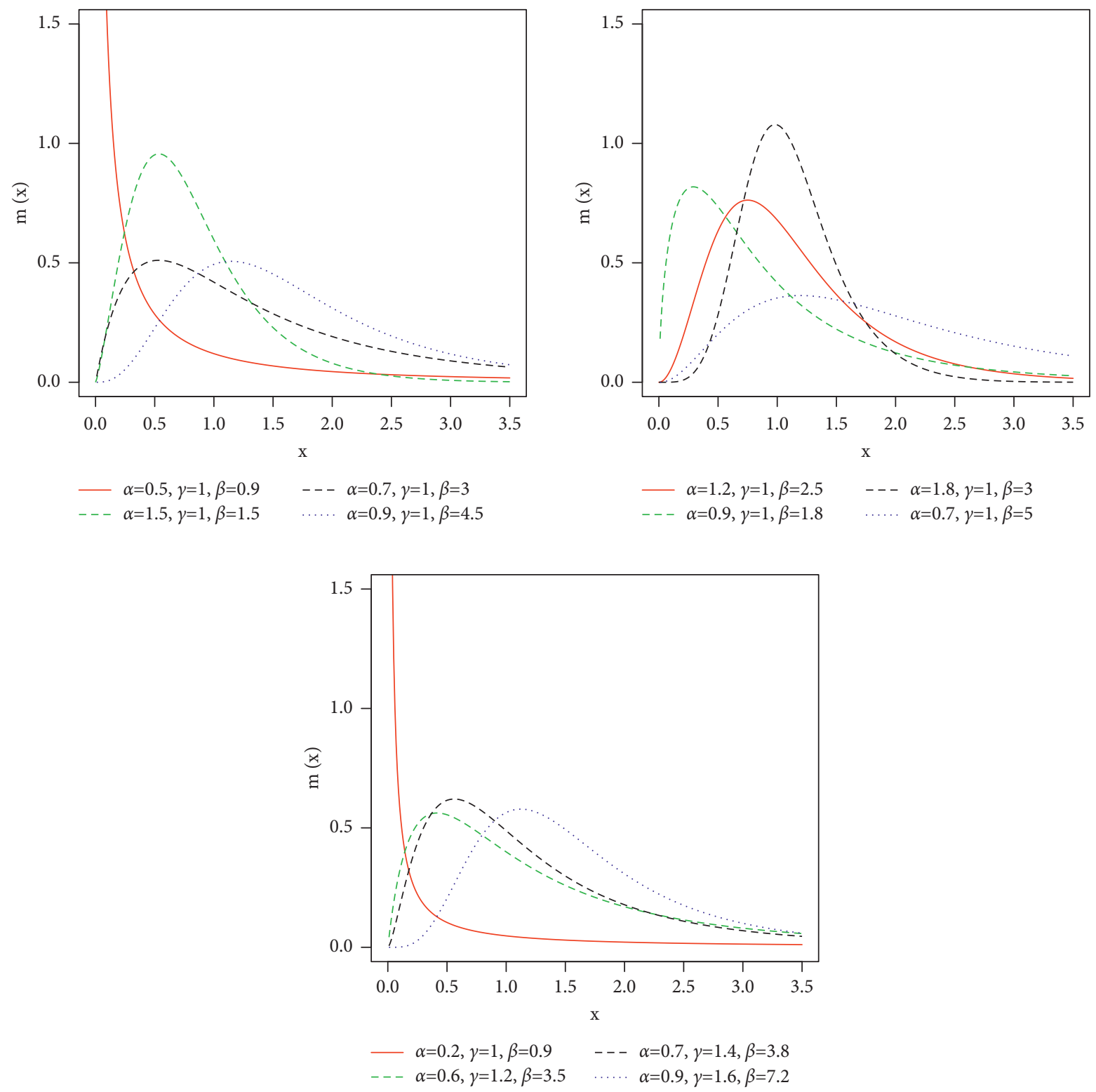

FIgURE 1: Visual display of $m(x ; \beta, \Delta)$.

$$
\begin{aligned}
\operatorname{bias}(\Lambda) & =\frac{1}{500} \sum_{i=1}^{500}(\widehat{\Lambda}-\Lambda), \\
\operatorname{MSE}(\Lambda) & =\frac{1}{500} \sum_{i=1}^{500}(\widehat{\Lambda}-\Lambda)^{2},
\end{aligned}
$$

where $\Lambda=(\beta, \Delta)$.

The summary measures (SMs) of the three simulated sets of data are provided in Table 1, whereas the histograms, box plots, kernel density estimator, and estimated CDF of the NE-Weibull model are provided in Figures 6-8. The simulation results are visually displayed in Figures 9-11.

\section{Data Modeling in the Finance Sector}

Here, we illustrate the importance of the NE-Weibull distribution in modeling insurance claims data from the financial sector. We also calculated the risk measures such as $\mathrm{VaR}$ (value at risk) and TVaR (tail value at risk) for this data.

6.1. Insurance Claims Data. The insurance claims data represents the initial claims of the unemployment insurances per month from 1971 to 2018 [20]. The data set can also be retrieved from https://data.worlddatany-govns $8 \mathrm{z}$ xewg. 

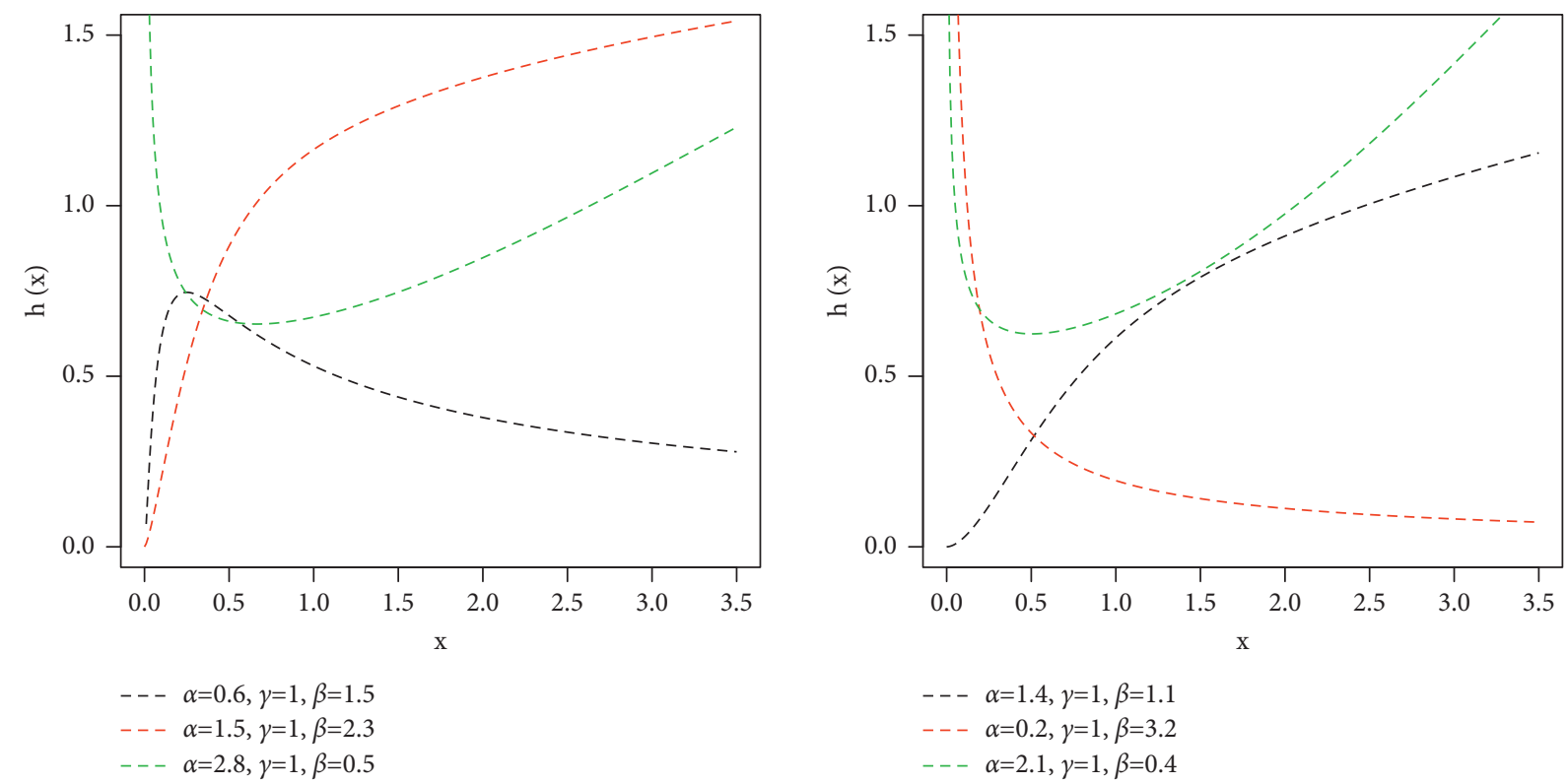

FiguRE 2: Different HRF plots of the NE-Weibull model.
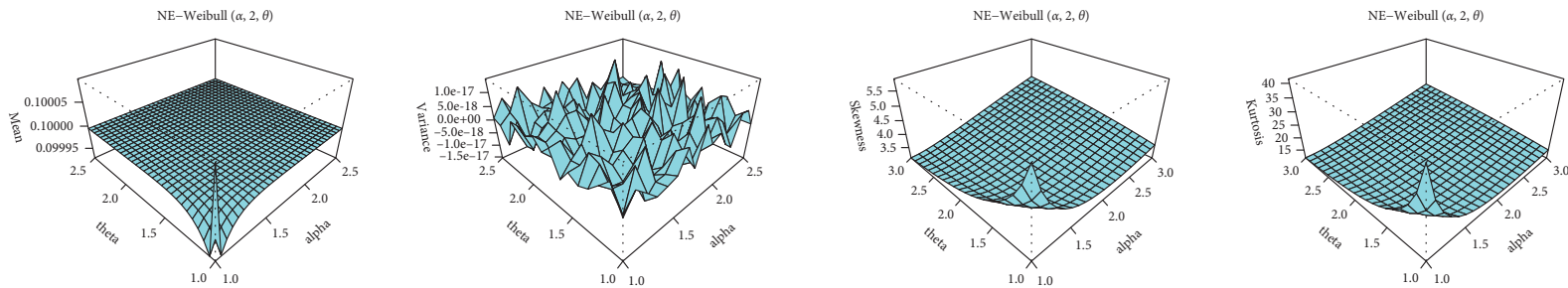

Figure 3: Plots of SK, KUR, variance, and mean of the NE-Weibull distribution for $\gamma=2$ and different values of $\alpha$ and $\beta$.
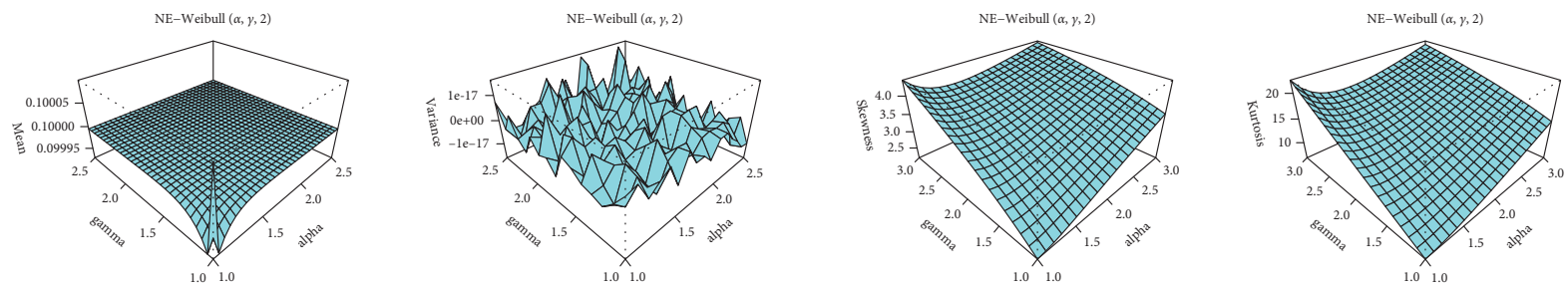

FIgURE 4: Plots of SK, KUR, variance, and mean of the NE-Weibull distribution for $\beta=2$ and different values of $\alpha$ and $\gamma$.

The summary statistics for the insurance claims data are reported in Table 2. Corresponding to this data, the histogram, box plot, and total time test (TTT) plots are provided in Figure 12. Figure 12 shows that the insurance claims data is unimodal, right-skewed, and HT.

The NE-Weibull distribution is applied to fit the financial data, and it is compared with the Weibull, W-Loss (WeibullLoss), NHT-Weibull (new heavy-tailed Weibull), Ku-Weibull (Kumaraswamy Weibull), and B-Weibull (beta Weibull) distributions. The CDFs of these models are as follows:

(i) Weibull model:

$$
H(x)=1-\exp \left\{-\gamma x^{\alpha}\right\}, \quad x \geq 0 .
$$

(ii) W-Loss model:

$$
H(x)=1-\frac{\beta \exp \left\{-\gamma x^{\alpha}\right\}}{\beta+\gamma x^{\alpha}}, \quad x \geq 0 .
$$

(iii) NHT-Weibull model:

$$
H(x)=\frac{\beta\left(1-\exp \left\{-\gamma x^{\alpha}\right\}\right)}{\left(\beta-\exp \left\{-\gamma x^{\alpha}\right\}\right)^{2}}, \quad x \geq 0 .
$$

(iv) $\mathrm{Ku}$-Weibull model:

$$
H(x)=1-\left[1-\left(1-\exp \left\{-\gamma x^{\alpha}\right\}\right)^{a}\right]^{b}, \quad x \geq 0 .
$$

(v) B-Weibull model:

$$
H(x)=I_{\left(1-e^{-\gamma x^{\alpha}}\right)}(x ; a, b), \quad x \geq 0 .
$$



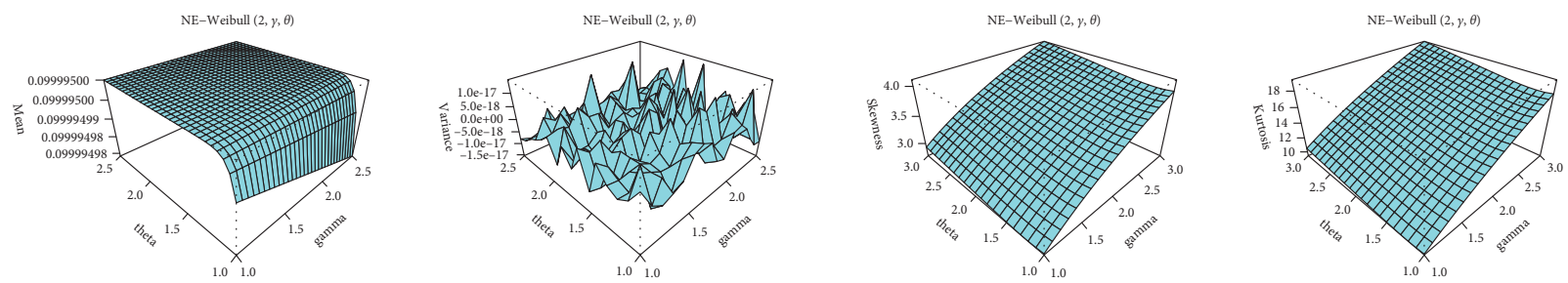

FIgURE 5: Plots of SK, KUR, variance, and mean of the NE-Weibull distribution for $\alpha=2$ and different values of $\beta$ and $\gamma$.

TABLE 1: SMs of the simulated data sets.

\begin{tabular}{lccccc}
\hline Simulated data & Min. & 1st Qu. & Median & Mean & 3rd Qu. \\
\hline Data set 1 & 0.0000 & 0.0390 & 0.1926 & 1.1191 & 0.9717 \\
Data set 2 & 0.0000 & 0.1388 & 0.3475 & 0.5469 & 0.7188 \\
Data set 3 & 0.0000 & 0.0070 & 0.0707 & 0.3364 & 0.3125 \\
\hline
\end{tabular}
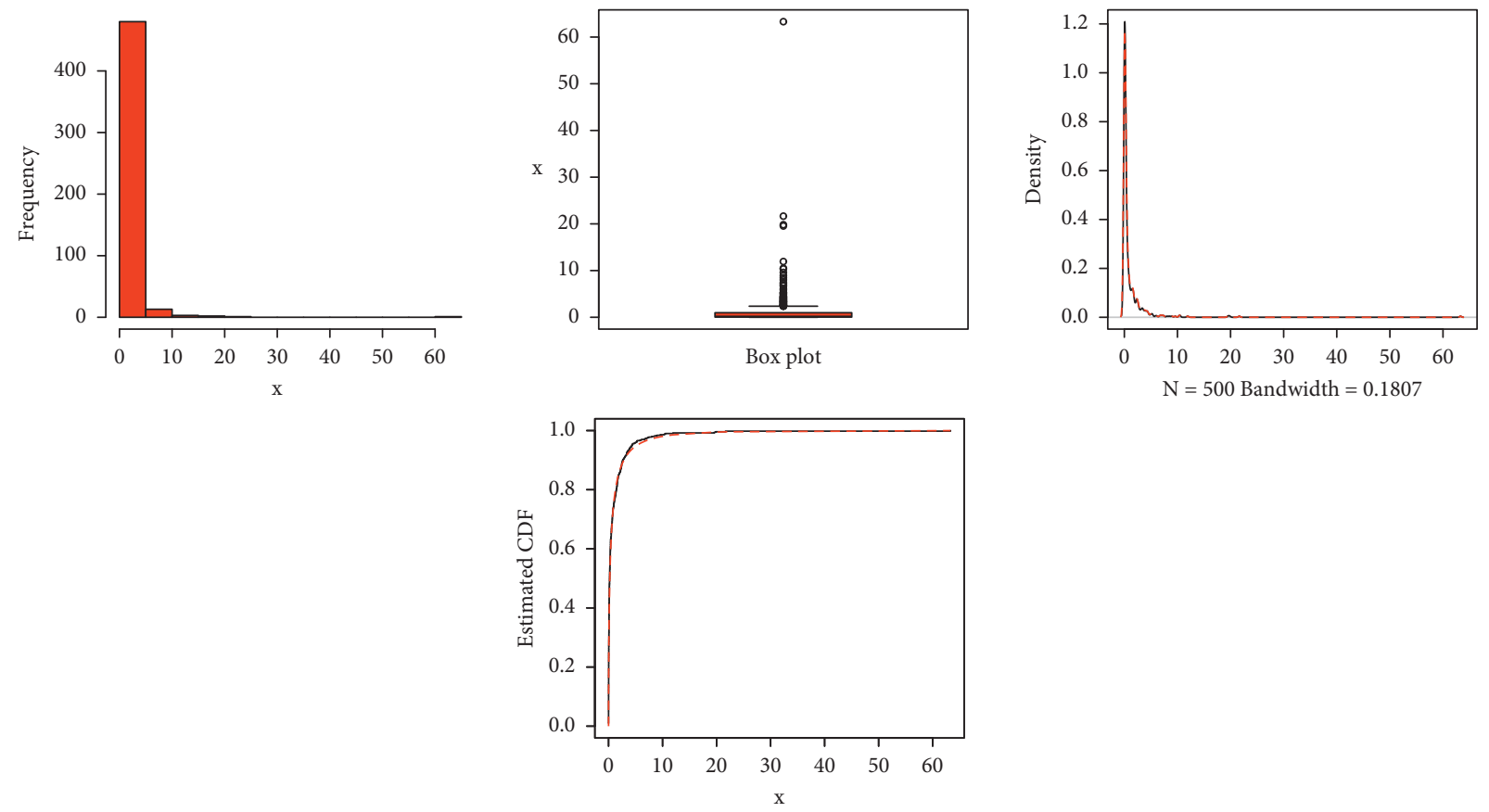

FIGURE 6: Kernel density estimator, box plot, histogram, and fitted CDF of the NE-Weibull distribution for simulated data set 1.

To decide about the best fitting of the applied distributions, certain criteria are taken into account. These criteria are as follows:

(i) The AIC is

$$
2 p-2 \ell(\boldsymbol{\Theta})
$$

(ii) The BIC is

$$
p \log (k)-2 \ell(\Theta)
$$

(iii) The HQIC is

$$
2 p \log (\log (k))-2 \ell(\boldsymbol{\Theta}) .
$$

(iv) The CAIC is

$$
\frac{2 p k}{k-p-1}-2 \ell(\Theta)
$$

Here, $\ell(\Theta)$ denotes the LL function, $\Theta$ is a parametric space, $p$ represents the model parameters, and $k$ is the number of selected samples. In addition to the criteria measures, three goodness-of-fit tests with its corresponding $p$ values are also considered. These tests are given by the following:

(i) The Anderson-Darling (AD) test statistic is

$$
\mathrm{AD}=-k-\frac{1}{n} \sum_{j=1}^{k}(2 j-1)\left[\log H\left(x_{j}\right)+\log \left\{1-H\left(x_{k-j+1}\right)\right\}\right],
$$



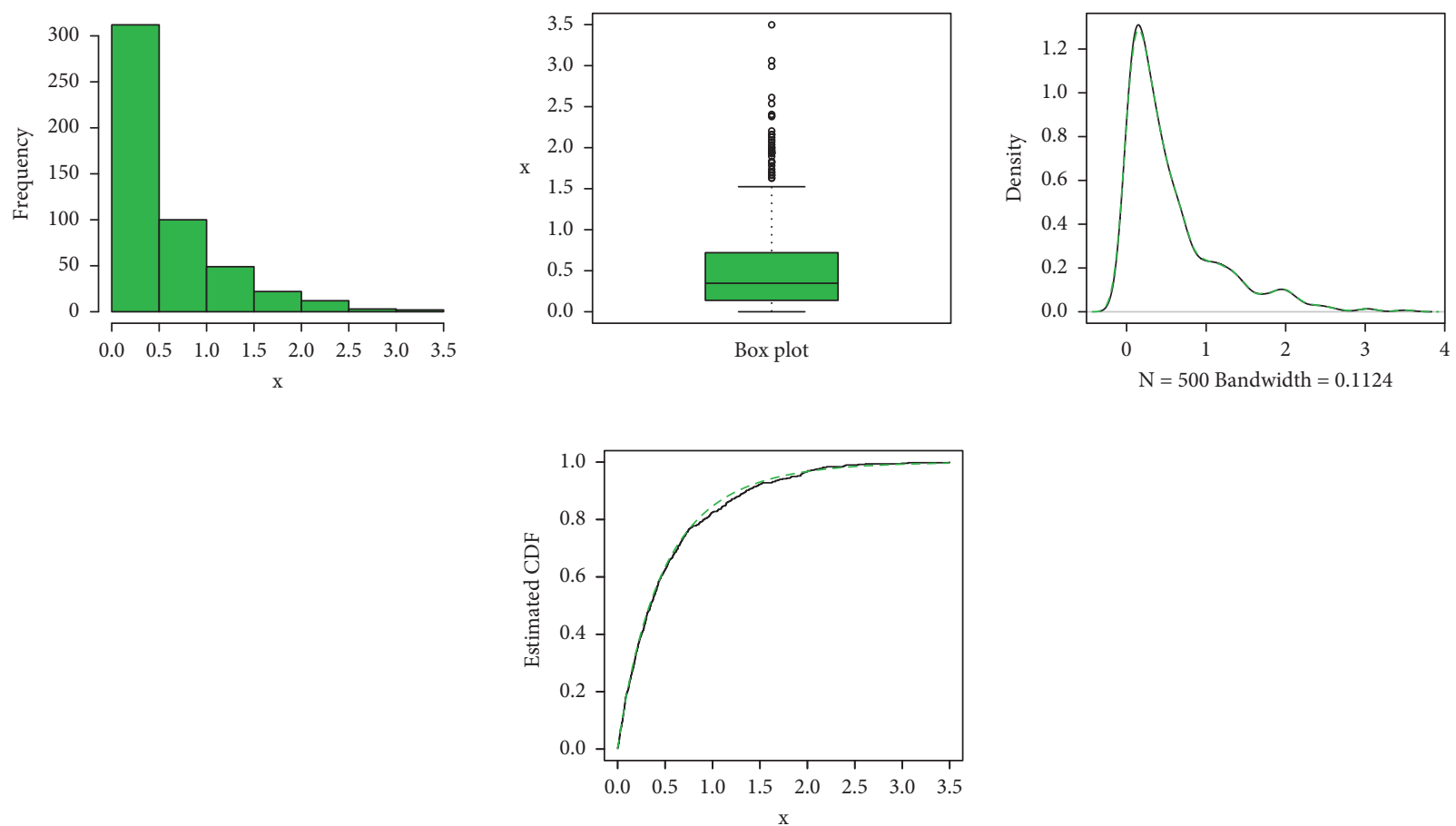

Figure 7: Kernel density estimator, box plot, histogram, and fitted CDF of the NE-Weibull distribution for simulated data set 2.
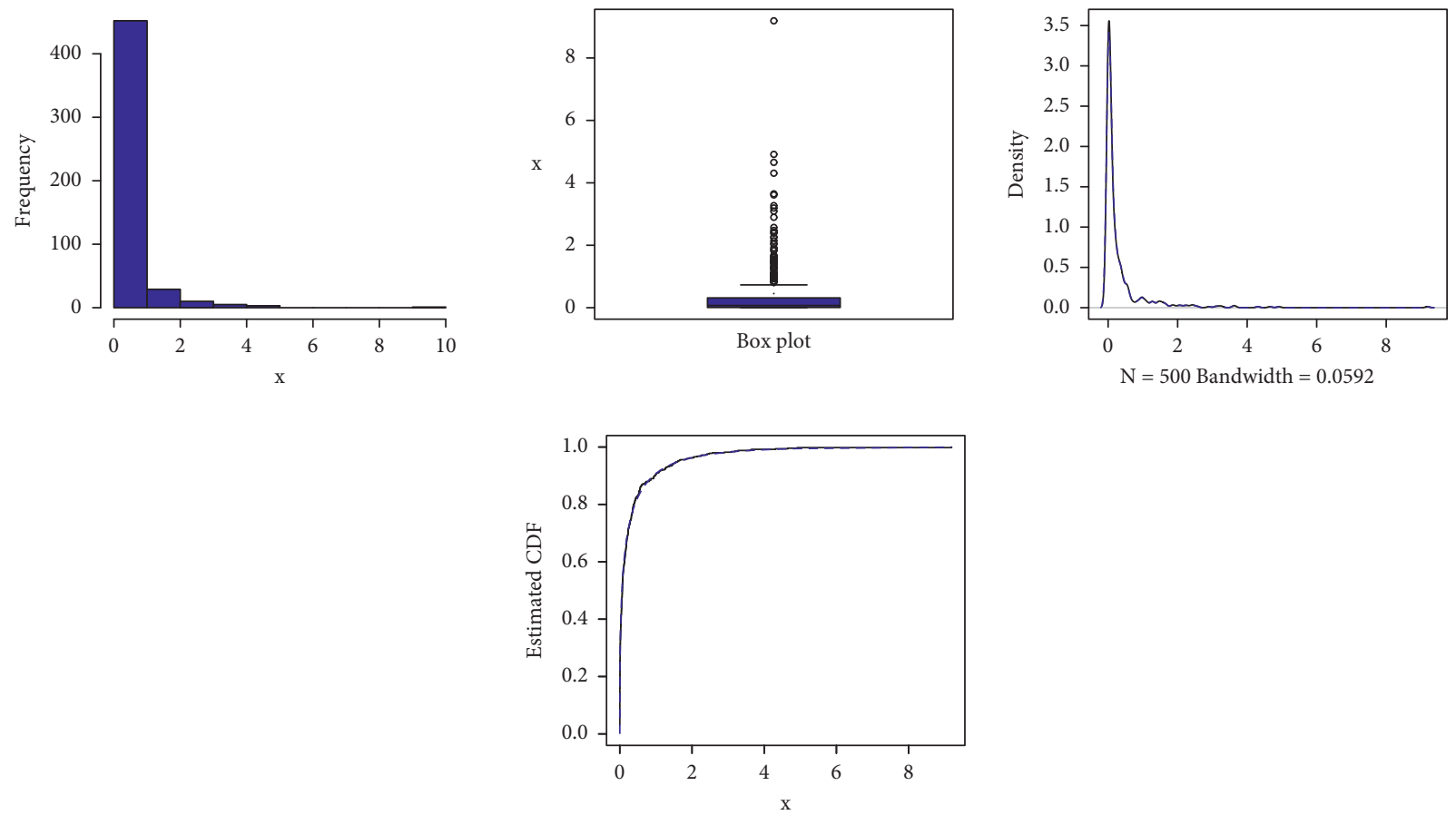

FIgURE 8: Kernel density estimator, box plot, histogram, and fitted CDF of the NE-Weibull distribution for simulated data set 3.

where $x_{j}$ is the $j^{\text {th }}$ observation in $k$ samples, calculated after sorting the data in the ascending order.

(ii) The Cramer-von Mises (CM) test statistic is

$$
\mathrm{CM}=\frac{1}{12 k}+\sum_{j=1}^{k}\left[\frac{2 j-1}{2 k}-H\left(x_{j}\right)\right]^{2} .
$$

(iii) The Kolmogorov-Smirnov (KS) test statistic is

$$
\mathrm{KS}=\sup _{x}\left[H_{n}(x)-H(x)\right],
$$

where $\sup _{x}$ represents the supremum of the set of distances and $H_{n}(x)$ indicates the empirical CDF. 

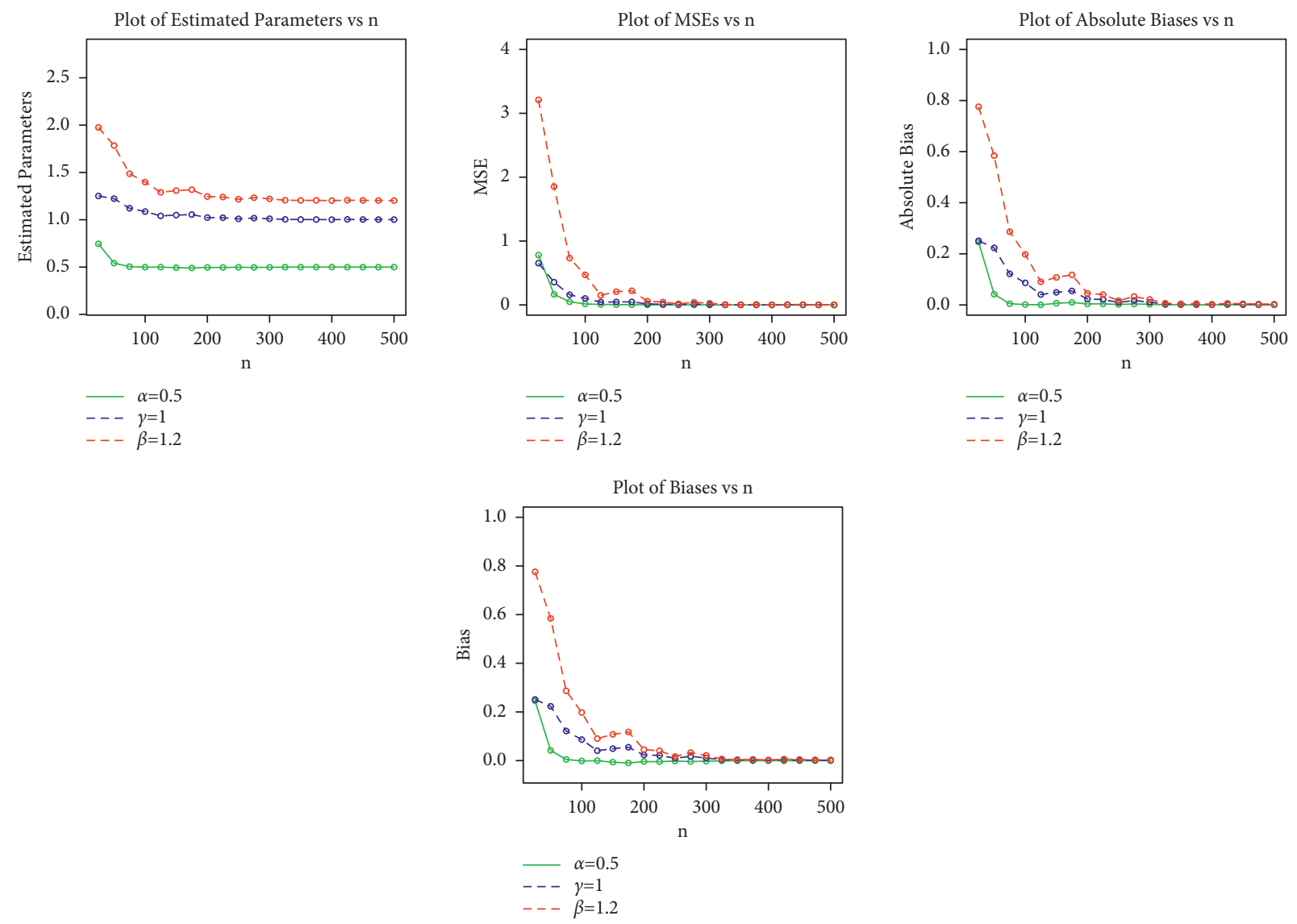

Figure 9: Graphical display of different measures for simulated data set 1.

Plot of Estimated Parameters vs n

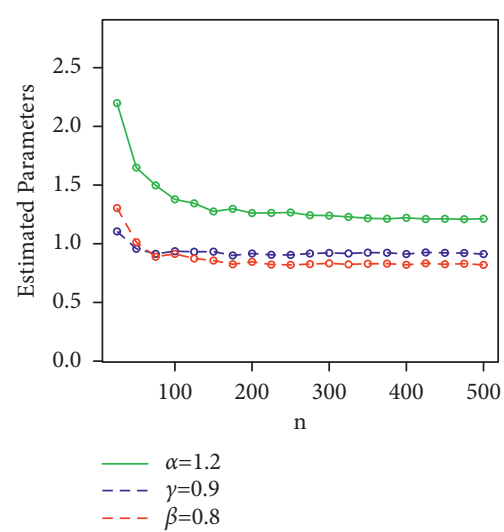

Plot of MSEs vs $n$

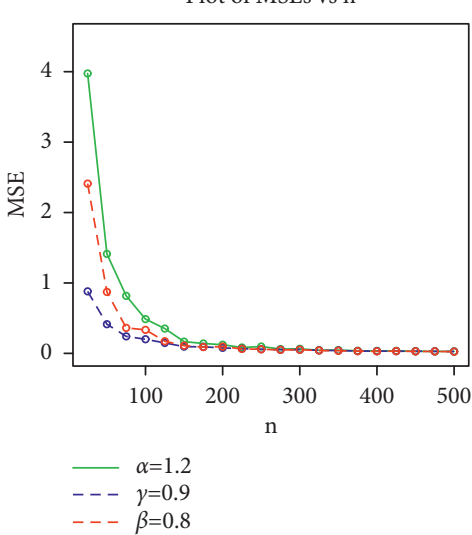

Plot of Biases vs n

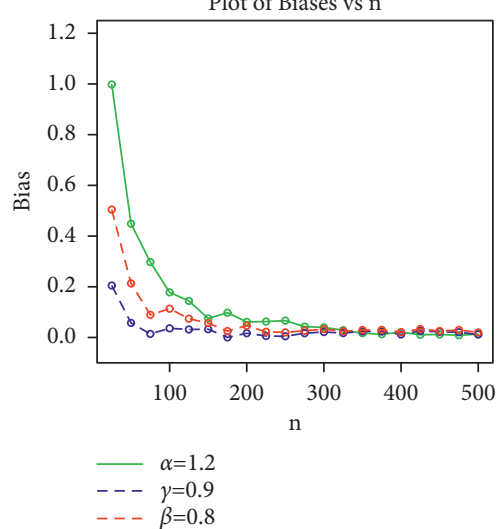

Plot of Absolute Biases vs n

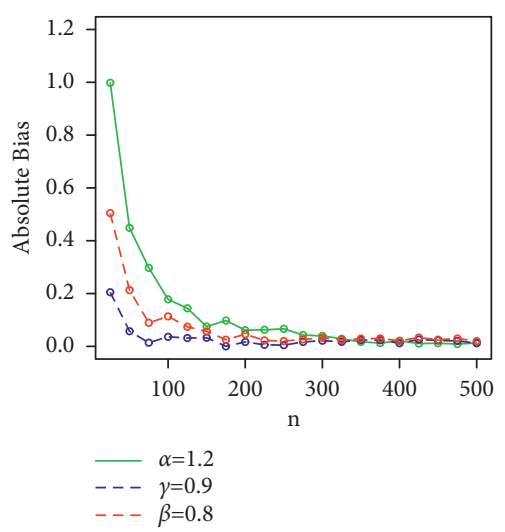

FIGURE 10: Graphical display of different measures for simulated data set 2. 

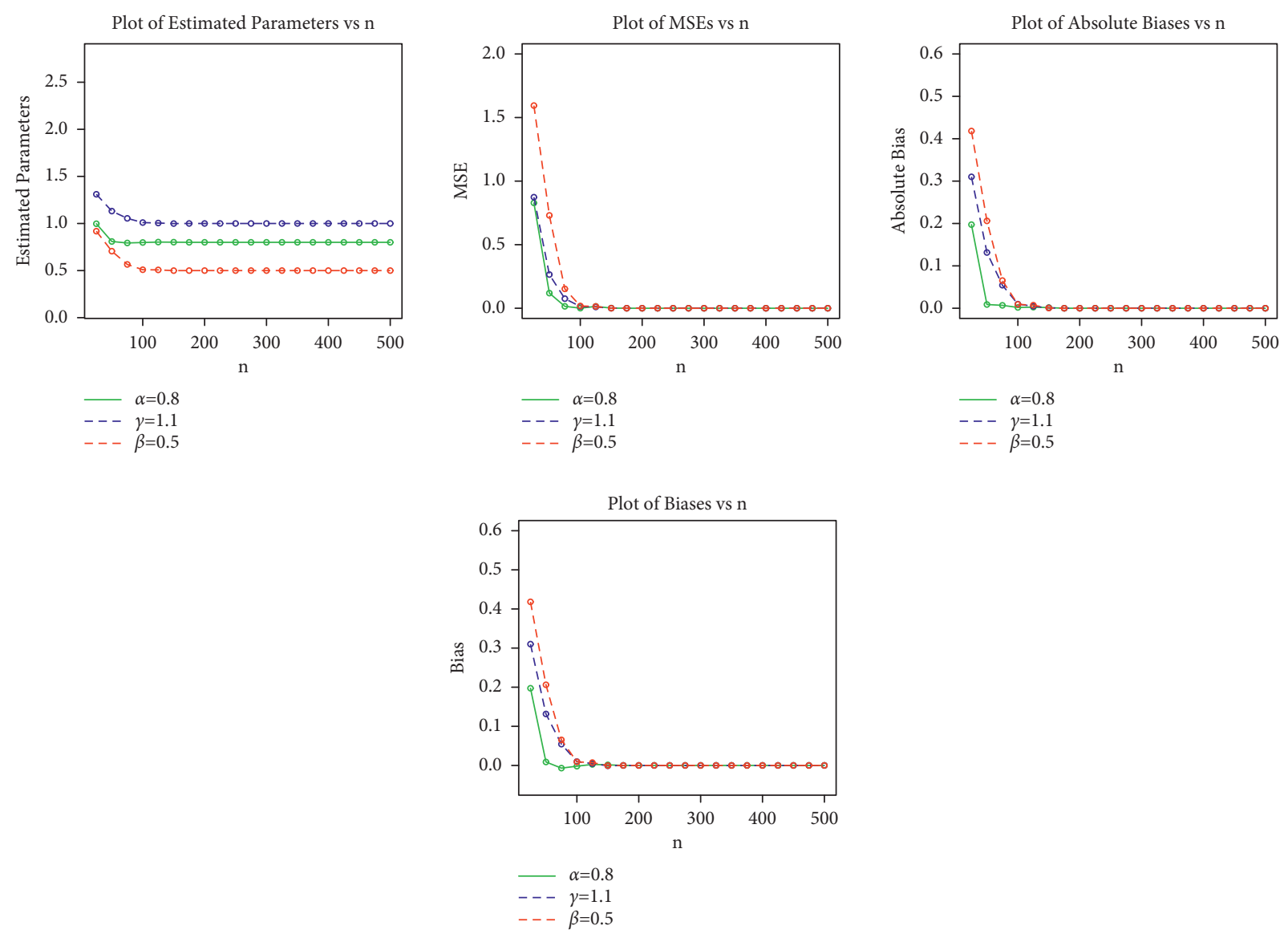

FIgURE 11: Graphical display of different measures for simulated data set 3.

TABLE 2: SMs of the insurance claims data.

\begin{tabular}{lccccc}
\hline Min. & 1st Qu. & Median & Mean & 3rd Qu. & Max. \\
\hline 49.26 & 82.71 & 100.02 & 109.18 & 129.74 & 308.35 \\
\hline
\end{tabular}
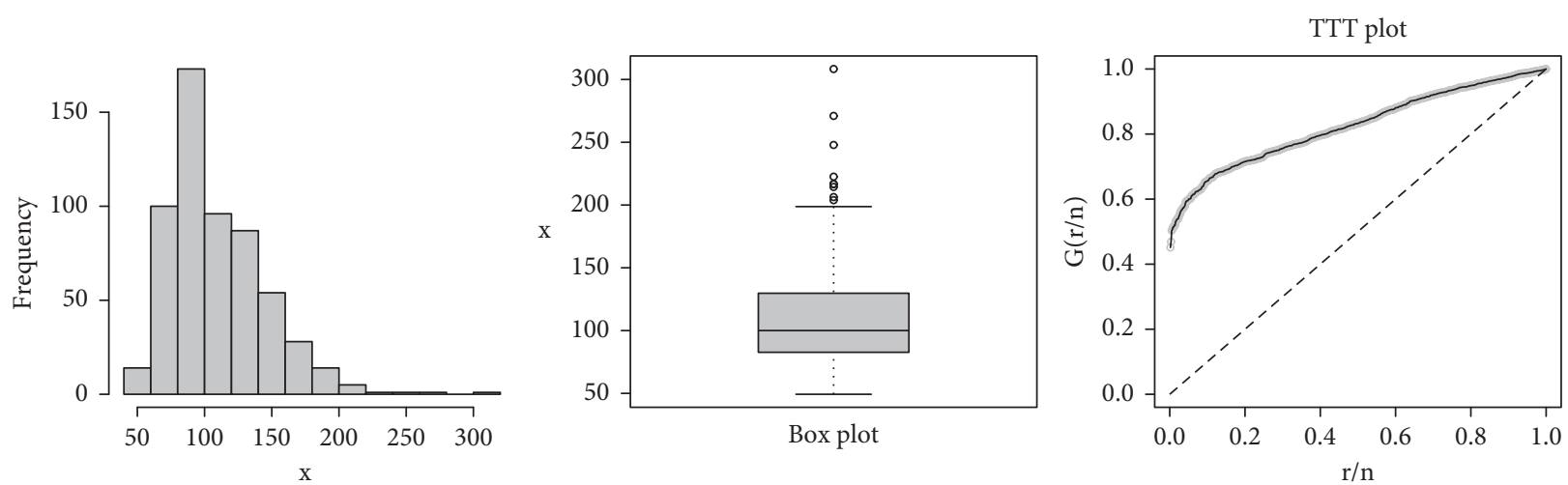

Figure 12: Histogram, TTT, and box plots for the insurance claims data.

The LL function is optimized, and the goodness-of-fit measures are calculated using the algorithm method "BFGS" via optim() R-function [31]. The MLEs of the fitted models with standard errors are listed in Table 3. Tables 4 and 5 show the goodness-of-fit values for the considered models. 
TABLE 3: Estimated parameters for the insurance claims data.

\begin{tabular}{|c|c|c|c|c|c|}
\hline Distribution & $\alpha$ & $\gamma$ & $\beta$ & $a$ & $b$ \\
\hline NE-Weibull & $1.283(0.012)$ & $0.005(0.001)$ & $8.689(0.610)$ & & \\
\hline Weibull & $1.486(1.096)$ & $0.001(0.095)$ & & & \\
\hline W-Loss & $1.564(1.075)$ & $0.041(0.076)$ & $12.765(0.963)$ & & \\
\hline NHT-Weibull & $1.316(0.964)$ & $0.025(0.049)$ & $10.498(0.875)$ & $31.713(3.791)$ & \\
\hline Ku-Weibull & $0.984(0.076)$ & $0.052(0.017)$ & & $64.933(0.063)$ & $0.657(0.167)$ \\
\hline B-Weibull & $1.090(0.058)$ & $0.031(0.000)$ & & $40.704(10.392)$ & $0.592(0.139)$ \\
\hline
\end{tabular}

Table 4: Discrimination criteria of the competitive models for the insurance claims data.

\begin{tabular}{lcccr}
\hline Distribution & AIC & BIC & CAIC & HQIC \\
\hline NE-Weibull & 5519.460 & 5532.523 & 5519.502 & 5524.555 \\
Weibull & 6169.574 & 6178.290 & 6169.634 & 6172.975 \\
W-Loss & 5585.738 & 5596.708 & 5585.984 & 5590.767 \\
NHT-Weibull & 5546.007 & 5564.756 & 5546.083 & 5553.865 \\
Ku-Weibull & 5601.734 & 5619.152 & 5601.805 & 5608.527 \\
B-Weibull & 5602.555 & 5602.625 & 5619.972 & 5609.348 \\
\hline
\end{tabular}

TABLE 5: Goodness-of-fit criteria of the competing distributions for insurance claims data.

\begin{tabular}{lcccc}
\hline Distribution & CM & AD & KS & $p$ value \\
\hline NE-Weibull & 0.146 & 0.674 & 0.016 & 0.668 \\
Weibull & 0.974 & 2.094 & 0.896 & 0.129 \\
W-Loss & 0.196 & 0.895 & 0.027 & 0.538 \\
NHT-Weibull & 0.175 & 0.795 & 0.023 & 0.618 \\
Ku-Weibull & 0.200 & 1.037 & 0.037 & 0.383 \\
B-Weibull & 0.221 & 1.121 & 0.035 & 0.471 \\
\hline
\end{tabular}
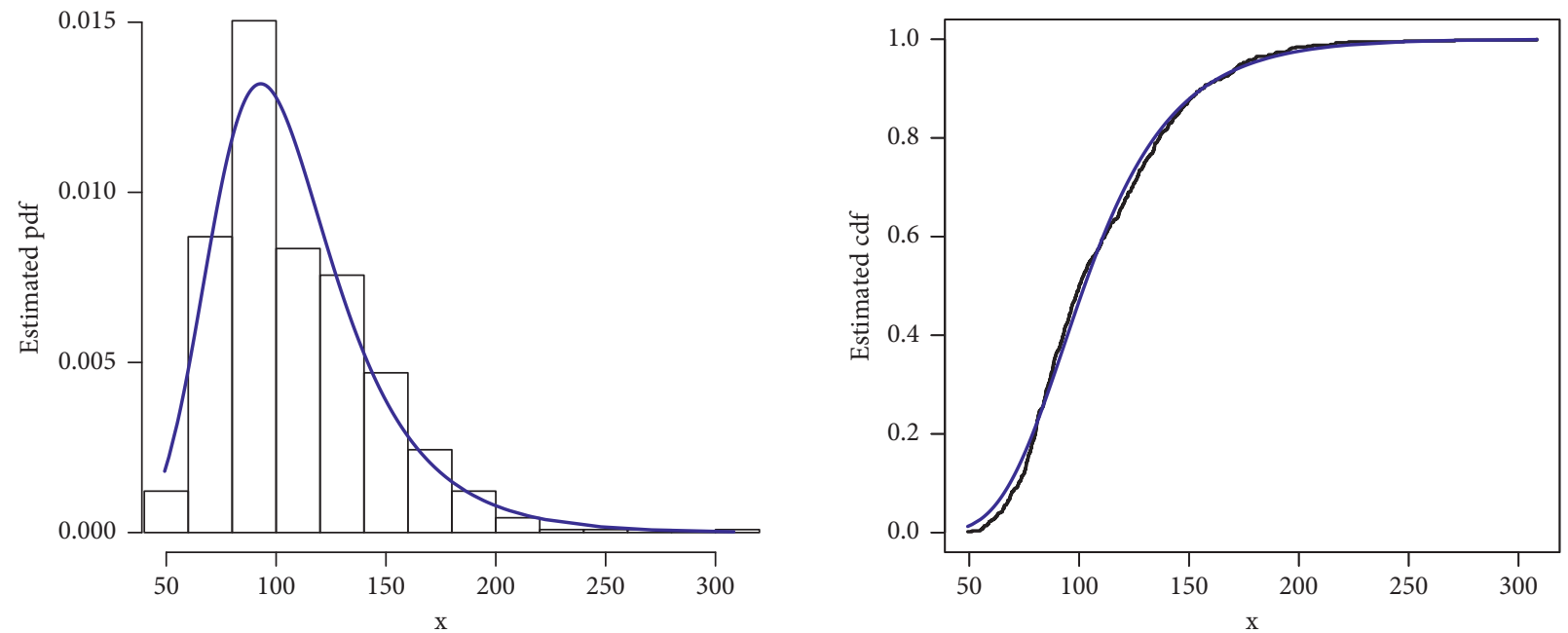

FIgURE 13: Estimated density and distribution functions of the NE-Weibull distribution.

The fitted PDF and CDF of the NE-Weibull model are provided in Figure 13, showing that the right-skewed HT data can be adequately fitted by the NE-Weibull distribution. The Kaplan-Meier survival (KMS) and PP (probability-probability) plots of the NE-Weibull distribution are sketched in Figure 14.

6.2. VaR and TVaR for Insurance Claims Data. Now, we calculate the VaR and TVaR measures for the Weibull and proposed NE-Weibull distributions based on the MLEs and the data provided in Section 6.1. The results of the VaR and TVaR of the NE-Weibull and Weibull distributions are presented in Table 6. We also display these results graphically in Figure 15.

A model having higher values for the TVaR and $\mathrm{VaR}$ is considered a HT distribution. The numerical results in Table 6 and their graph in Figure 15 show that the NEWeibull is a HT model. 

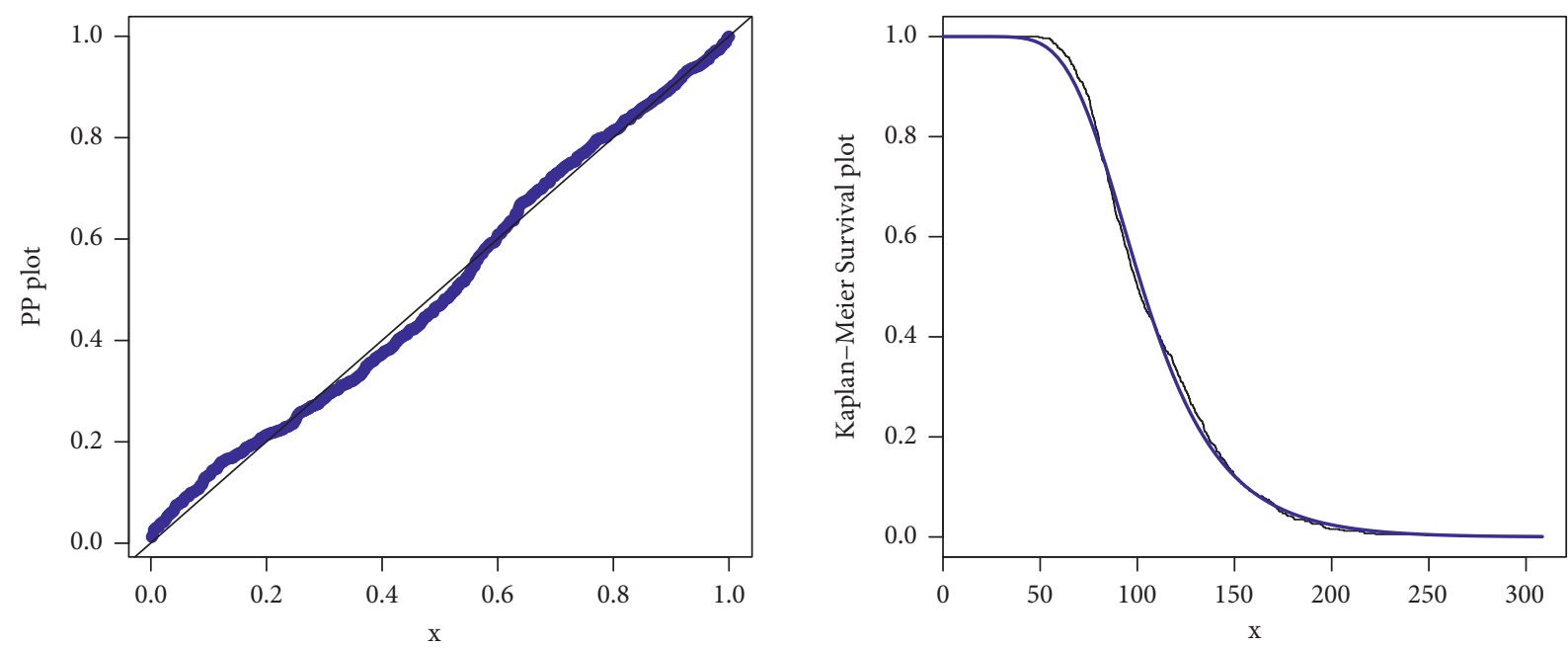

FIgURE 14: KMS and PP plots of the NE-Weibull distribution.

TABLe 6: VaR and TVaR results for the NE-Weibull and Weibull distributions.

\begin{tabular}{|c|c|c|c|c|}
\hline Distribution & Parameters & Level of significance & $\mathrm{VaR}$ & TVaR \\
\hline \multirow{8}{*}{ NE-Weibull } & \multirow{8}{*}{$\begin{array}{l}\alpha=1.283 \\
\gamma=0.005 \\
\beta=8.689\end{array}$} & 0.700 & 145.8537 & 153.9659 \\
\hline & & 0.750 & 153.5376 & 179.2175 \\
\hline & & 0.800 & 168.5360 & 198.7654 \\
\hline & & 0.850 & 192.3638 & 218.7189 \\
\hline & & 0.900 & 209.8965 & 231.9876 \\
\hline & & 0.950 & 224.0768 & 249.0786 \\
\hline & & 0.975 & 239.7853 & 268.0298 \\
\hline & & 0.999 & 253.6358 & 287.0948 \\
\hline \multirow{8}{*}{ Weibull } & \multirow{8}{*}{$\begin{array}{l}\alpha=1.486 \\
\gamma=0.001\end{array}$} & 0.700 & 70.3462 & 89.7568 \\
\hline & & 0.750 & 77.7643 & 101.8539 \\
\hline & & 0.800 & 83.8743 & 115.7943 \\
\hline & & 0.850 & 98.3245 & 135.8764 \\
\hline & & 0.900 & 120.5643 & 156.9875 \\
\hline & & 0.950 & 142.6750 & 179.9876 \\
\hline & & 0.975 & 176.7656 & 201.9875 \\
\hline & & 0.999 & 202.6752 & 230.9786 \\
\hline
\end{tabular}

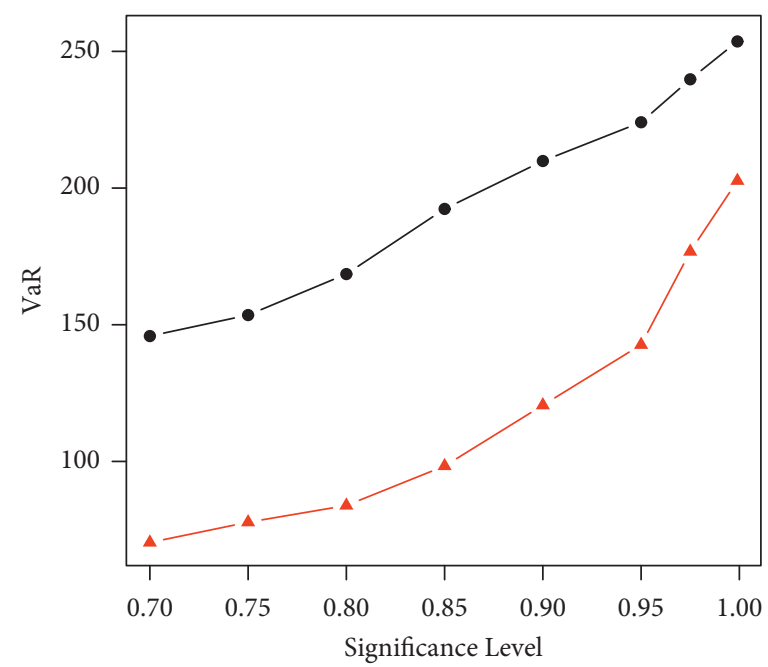

$\Delta$ Weibull

- NE-Weibull

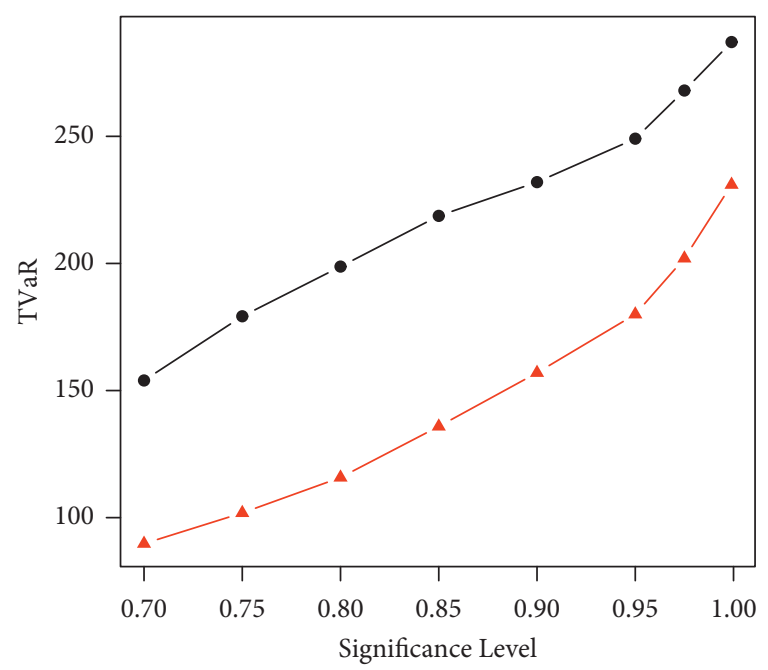

A Weibull

- NE-Weibull

FIgURE 15: Graphical presentation for the values of the VaR and TVaR. 


\section{Final Comments}

During the last couple of years, the study and modeling of extreme value data has gained increased interest in numerous areas, particularly in the hydrology and finance sector. Recent studies have shown the importance and potential of the HT distributions in various areas for analyzing real data sets, especially in the finance sector. In this article, a new interesting HT extension of the Weibull distribution, called the NE-Weibull distribution, is proposed using the NE- $X$ approach. The MLEs of the NE-Weibull parameters $\beta$ and $\Delta$ are obtained. To show the applicability of the NE-Weibull model, a real-life insurance claims data from the financial sector is considered. Based on certain tests, it is showed that the NE-Weibull model offers an adequate fit to the insurance claims data than the Weibull, W-Loss, NHT-Weibull, Ku-Weibull, and B-Weibull. On the other hand, the numerical results for the VaR and TVaR measures showed that the NE-Weibull model is a HT distribution as compared to the classical Weibull distribution.

\section{Data Availability}

To replicate the findings of this paper, the data is available from the corresponding author upon request.

\section{Disclosure}

This article was drafted from the $\mathrm{PhD}$ work of the first author (Zubair Ahmad).

\section{Conflicts of Interest}

The authors declare no conflicts of interest regarding the publication of this work.

\section{Authors' Contributions}

Conceptualization and methodology were carried out by Zubair Ahmad and Eisa Mahmoudi. The original draft was prepared by Zubair Ahmad and Morad Alizadeh. The original draft was edited by Eisa Mahmoudi, Rasool Roozegar, and Ahmed Z. Afify. Formal analysis was performed by Zubair Ahmad. Software was collected by Zubair Ahmad and Morad Alizadeh. The study was supervised by Eisa Mahmoudi, Morad Alizadeh, and Rasool Roozegar. Investigation was conducted by Zubair Ahmad. The manuscript was reviewed and edited by Zubair Ahmad, Eisa Mahmoudi, Morad Alizadeh, Rasool Roozegar, and Ahmed Z. Afify.

\section{Acknowledgments}

This work was supported by the Department of Statistics, Yazd University, Yazd, Iran.

\section{References}

[1] M. N. Lane, "Pricing risk transfer transactions," ASTIN Bulletin, vol. 30, no. 2, pp. 259-293, 2000.
[2] K. Cooray and M. Ananda, "Modeling actuarial data with a composite lognormal-Pareto model," Scandinavian Actuarial Journal, vol. 2005, no. 5, pp. 321-334, 2005.

[3] H. J. Wang, D. Li, and X. He, "Estimation of high conditional quantiles for heavy-tailed distributions," Journal of the American Statistical Association, vol. 107, no. 500, pp. 14531464, 2012.

[4] S. Ahn, J. H. T. Kim, and V. Ramaswami, "A new class of models for heavy tailed distributions in finance and insurance risk," Insurance: Mathematics and Economics, vol. 51, no. 1, pp. 43-52, 2012.

[5] P. R. Jelenković and J. Tan, "Characterizing heavy-tailed distributions induced by retransmissions," Advances in Applied Probability, vol. 45, no. 1, pp. 106-138, 2013.

[6] F. Forbes and D. Wraith, "A new family of multivariate heavytailed distributions with variable marginal amounts of tailweight: application to robust clustering," Statistics and Computing, vol. 24, no. 6, pp. 971-984, 2014.

[7] Z. Guo, "Heavy-tailed distribution and risk management of equity market tail events," Journal of Risk \& Control, vol. 4, no. 1, pp. 31-41, 2017.

[8] A. Punzo, L. Bagnato, and A. Maruotti, "Compound unimodal distributions for insurance losses," Insurance: Mathematics and Economics, vol. 81, pp. 95-107, 2018.

[9] D. Bhati and S. Ravi, "On generalized log-Moyal distribution: a new heavy tailed size distribution," Insurance: Mathematics and Economics, vol. 79, pp. 247-259, 2018.

[10] A. Punzo, "A new look at the inverse Gaussian distribution with applications to insurance and economic data," Journal of Applied Statistics, vol. 46, no. 7, pp. 1260-1287, 2019.

[11] Y. Ke, S. Minsker, Z. Ren, Q. Sun, and W. X. Zhou, "Userfriendly covariance estimation for heavy-tailed distributions," Statistical Science, vol. 34, no. 3, pp. 454-471, 2019.

[12] E. F. Dos Reis, A. Li, and N. Masuda, "Generative models of simultaneously heavy-tailed distributions of interevent times on nodes and edges," Physical Review E, vol. 102, no. 5, Article ID 052303, 2020.

[13] H. Niu, J. Wei, and Y. Chen, "Optimal randomness for stochastic configuration network (SCN) with heavy-tailed distributions," Entropy, vol. 23, no. 1, p. 56, 2021.

[14] M. Bladt, B. F. Nielsen, and G. Samorodnitsky, "Calculation of ruin probabilities for a dense class of heavy tailed distributions," Scandinavian Actuarial Journal, vol. 2015, no. 7, pp. 573-591, 2015.

[15] K. Tikhomirov, "Sample covariance matrices of heavy-tailed distributions," International Mathematics Research Notices, vol. 2018, no. 20, pp. 6254-6289, 2018.

[16] G. Lugosi and S. Mendelson, "Mean estimation and regression under heavy-tailed distributions: a survey," Foundations of Computational Mathematics, vol. 19, no. 5, pp. 1145-1190, 2019.

[17] D. Yousri, M. Abd Elaziz, L. Abualigah, D. Oliva, M. A. A. AlQaness, and A. A. Ewees, "COVID-19 X-ray images classification based on enhanced fractional-order cuckoo search optimizer using heavy-tailed distributions," Applied Soft Computing, vol. 101, Article ID 107052, 2021.

[18] P. L. Ramos, D. C. Nascimento, C. Cocolo et al., "Reliabilitycentered maintenance: analyzing failure in harvest sugarcane machine using some generalizations of the Weibull distribution," Modelling and Simulation in Engineering, vol. 2018, Article ID 1241856, 12 pages, 2018.

[19] E. Ramos, P. L. Ramos, and F. Louzada, "Posterior properties of the Weibull distribution for censored data," Statistics \& Probability Letters, vol. 166, Article ID 108873, 2020. 
[20] W. He, Z. Ahmad, A. Z. Afify, and H. Goual, "The arcsine exponentiated-X family: validation and insurance application," Complexity, vol. 2020, Article ID 8394815, 18 pages, 2020.

[21] N. M. Alfaer, A. M. Gemeay, H. M. Aljohani, and A. Z. Afify, "The extended log-logistic distribution: inference and actuarial applications," Mathematics, vol. 9, no. 12, p. 1386, 2021.

[22] A. Z. Afify, G. M. Cordeiro, N. A. Ibrahim, F. Jamal, M. Elgarhy, and M. A. Nasir, "The Marshall-Olkin odd Burr III-G family: theory, estimation, and engineering applications," IEEE Access, vol. 9, pp. 4376-4387, 2021.

[23] A. Al Mutairi, M. Z. Iqbal, M. Z. Arshad, B. Alnssyan, H. AlMofleh, and A. Z. Afify, "A new extended model with bathtubshaped failure rate: properties, inference, simulation, and applications," Mathematics, vol. 9, no. 17, p. 2024, 2021.

[24] S. I. Resnick, "Discussion of the Danish data on large fire insurance losses,” ASTIN Bulletin, vol. 27, no. 1, pp. 139-151, 1997.

[25] J. Beirlant, G. Matthys, and G. Dierckx, "Heavy-tailed distributions and rating," ASTIN Bulletin, vol. 31, no. 1, pp. 37-58, 2001.

[26] S. Nadarajah and S. A. A. Bakar, "New composite models for the Danish fire insurance data," Scandinavian Actuarial Journal, vol. 2014, no. 2, pp. 180-187, 2014.

[27] Z. Ahmad, E. Mahmoudi, M. Alizadeh, R. Roozegar, and A. Z. Afify, "The exponential TX family of distributions: properties and an application to insurance data," Journal of Mathematics, vol. 2021, Article ID 3058170, 18 pages, 2021.

[28] A. Alzaatreh, C. Lee, and F. Famoye, "A new method for generating families of continuous distributions," Metron, vol. 71, no. 1, pp. 63-79, 2013.

[29] Z. Ahmad, G. G. Hamedani, and N. S. Butt, "Recent developments in distribution theory: a brief survey and some new generalized classes of distributions," Pakistan Journal of Statistics and Operation Research, vol. XV, pp. 87-110, 2019.

[30] F. Jamal and M. Nasir, "Some new members of the TX family of distributions," in Proceedings of the 17th International Conference on Statistical Sciences, vol. 33, Trieste, Italy, July 2019.

[31] R. C. Team, R: A Language and Environment for Statistical Computing, R Foundation for Statistical Computing, Vienna, Austria, 2019, https://www.Rproject.org/ Version 3.5.2. 